\title{
Mode Structure of Active Resonators
}

\author{
G. J. ERNST AND W. J. WITTEMAN
}

\begin{abstract}
An analysis is made of the mode structure of lasers when the interaction with the active medium is taken into account. We consider the combined effect of gain and refractive-index variations for arbitrary mirror configurations. Using a dimensionless round-trip matrix for a medium with a quadratic variation of the propagation constant with distance from the optic axis, dimensionless beam parameters are derived in terms of cavity and medium parameters. Beam waist and radius of curvature are obtained explicitly as a function of a dimensionless parameter containing the variation of propagation constant, wavelength, and cavity length. The spot size depends only on the absolute value of the gain variation, whereas the deviation of the radius of curvature of the beam from the mirror curvature changes sign when the sign of the gain variation is reversed. As long as radial gain variations are present stable oscillations can always be obtained for any set of curved and/or flat mirrors. For large gain variations the spot size and radius of curvature of the outcoming beam become independent of mirror curvatures.
\end{abstract}

\section{INTRODUCTION}

$\mathbf{I}^{N}$ $\mathrm{N}$ designing laser cavities some problems are optical performance, such as stability of mode structure, maximizing output power, single-mode operation, and description of mode pattern. All these problems are interrelated and depend on the geometry of the cavity and plasma parameters of the active medium. In many cases the problems are simplified by considering only the geometry of the cavity and not taking into account the interaction with the active medium. This is done in the wellknown theory of Kogelnik and Li [1]. Such a treatment is justified since in most practical cases of small cavities having high quality factor and low output power, the influence of the active medium is negligible, in fact, it is well known that the observed mode patterns of such lasers do not show any perceptible deviation from modes predicted on the basis of the empty-space resonator theory.

However, these simplifications are less valid for increasing length of laser tube, increasing output power, and/or increasing transmission of outcoupling mirror. The theory eventually breaks down. Experimentally, it is observed that the optical performance of, for instance, a $\mathrm{CW} \mathrm{CO}_{2}$ laser of 4-m length cannot be described by the empty-space resonator theory. As we shall show in this paper, the beam waist of the Gaussian mode deviates considerably from predictions of empty-space theory. The experiments indicate that the influence of nonuniform active-medium parameters and outcoupling have to be included in the mode theory.

Several physical processes within the active medium can be studied in relation to wave propagation in optical

Manuscript received February 19, 1973; revised May 11, 1973.

The authors are with the Physics Department, Twente University of Technology, Enschede, The Netherlands. resonators. Comparing the estimated order of magnitude of their influence on the mode properties, it turns out that the most important ones with respect to beam propagation are heat dissipation within the gas discharge (variation of refractive index), inhomogeneous gain distribution [2], and the dispersion of the active medium [3]. These interactions are, in most configurations, axially symmetric due to either the excitation mechanism or the mutual interaction of medium and axially symmetric field. For example, the propagation of a Gaussian beam through a homogeneously excitated active medium results in axially symmetric gain variations.

The interaction can always be described in terms of the propagation constant (real and imaginary part) which may then be used for calculating the change of laser parameters. It has been shown by the theoretical work of Kogelnik [4] that, if the propagation constant varies only quadratically with distance from the axis, a Gaussian beam remains Gaussian while propagating through such a medium. This result may explain why in actual resonators Gaussian distributions are still observed.

In the present work we shall use Kogelnik's [4] result as our starting point for a theoretical evaluation of beam parameters of a laser system with arbitrary mirror curvatures and with a quadratic gain and index profile for the active medium. Although an exact quadratic profile will not easily be realized in practice, many actual systems can be described approximately by such a profile. Hence, this approach will give an improved description of laser systems over the empty-space theory. In fact, an experimental example presented below can be understood by using this approach.

The present theoretical results lead to new interesting conclusions concerning stability of resonator configuration, effect of gain variation on beam waist, curvature of wavefront, etc. Some conclusions are conflicting with previously published results.

Although, as we mentioned, in experimental systems the variations of propagation constants are not exactly quadratic, the main terms near the optical axis, due to gain variations, dispersion, and heat effects, are quadratic. Thus our theoretical results can be applied to actual systems.

\section{General Theory on the Mode Structure for a Nonlinear Medium}

For paraxial Gaussian beams [1] the beam width or spot size $\omega$ which measures the distance at which the field amplitude is $1 / e$ times that on the axis, and the radius of 
curvature $R$ are closely related and can be conveniently combined to a complex beam parameter $q$ defined by

$$
\frac{1}{q(z)}=\frac{1}{R(z)}-i \frac{\lambda}{\pi \omega^{2}(z)}
$$

where $z$ is the direction of propagation and $\lambda$ the wavelength in the medium. $R(z)$ is defined positive for a diverging beam. Tracing a Gaussian beam through a lens or lenslike medium the complex beam parameter $q$ obeys the so called $A B C D$ law

$$
q_{2}=\frac{A q_{1}+B}{C q_{1}+D}
$$

where $q_{1}$ and $q_{2}$ are the beam parameters at two reference planes. $A, B, C$, and $D$ are matrix elements describing the optical system.

In the following we apply Kogelnik's $A B C D$ law (2) to calculate a complete round trip through a resonator of length $L$ and with mirrors with radius of curvature $R_{1}$ and $R_{2}$. However, before doing this, we introduce dimensionless quantities for the matrix elements as well as for the beam parameters. The results are of course not different, but the advantage of this, as we shall see, will be that the beam waist and radius of curvature are explicitly obtained as a function of one or two dimensionless parameters that contain the nonlinearity of the medium, the wavelength, and the cavity length. The derivation thus is general without specifying explicitly all quantities like cavity length, mirror curvatures, wavelength, or quadratic terms of the active medium. Hence, the results are comprehensive and applicable to any laser resonator and wavelength.

The complex dimensionless beam parameters $P(z)$ is

$$
P(z)=U(z)-i Q(z)
$$

where

$$
U(z)=L / R(z)
$$

and formally the same as those found for the paraxial rays, except that one has, in general, a complex refractive index. Deriving this transfer matrix in dimensionless form we obtain

$$
\left|\begin{array}{ll}
e & f \\
g & h
\end{array}\right|=\left(\begin{array}{cc}
\cos \sqrt{\frac{k_{2}}{k_{0}}} L & \frac{1}{L} \sqrt{\frac{k_{0}}{k_{2}}} \sin \sqrt{\frac{k_{2}}{k_{0}}} L \\
-L \sqrt{\frac{k_{2}}{k_{0}}} \sin \sqrt{\frac{k_{2}}{k_{0}}} L & \cos \sqrt{\frac{k_{2}}{k_{0}}} L
\end{array}\right)
$$

where we have used for the propagation constant $k$ the form

$$
k=k_{0}-{ }_{2}^{1} k_{2} r^{2}
$$

with constants $k_{0}$ and $k_{2}$.

The dimensionless matrix corresponding to a complete round trip starting at $z=0$ through a resonator defined by two mirrors with radius of curvature $R_{1}$ and $R_{2}$ at $z=0$ and $z=L$, respectively, is then given by

$$
\begin{aligned}
& \left(\begin{array}{ll}
a_{0} & b_{0} \\
c_{0} & d_{0}
\end{array}\right)=\left(\begin{array}{cc}
1 & 0 \\
-2 U_{1} & 1
\end{array}\right)\left(\left[\begin{array}{ll}
e & f \\
g & h
\end{array}\right)\left(\begin{array}{cc}
1 & 0 \\
-2 U_{2} & 1
\end{array}\right)\left[\begin{array}{ll}
e & f \\
g & h
\end{array}\right)\right. \\
& =\left\{\begin{array}{c}
e^{2}-2 e f U_{2}+f g \\
-2 e^{2} U_{1}+e g+\left(h-2 f U_{1}\right)\left(g-2 e U_{2}\right)
\end{array}\right. \\
& \left.\begin{array}{c}
e f-2 f^{2} U_{2}+f h \\
-2 e f U_{1}+f g+\left(h-2 f U_{1}\right)\left(h-2 f U_{2}\right)
\end{array}\right)
\end{aligned}
$$

where $U_{1}=L / R_{1}$ and $U_{2}=L / R_{2}$. The values of $U_{1}$ or $U_{2}$ are positive if the reflected beam is made convergent; otherwise, they are negative.

Starting at $z=0$ with $P_{0}$, the $P$ parameter after a full round trip becomes

$$
P_{2}=\frac{c_{0}+d_{0} P_{0}}{a_{0}+b_{0} P_{0}}
$$

In order to obtain the steady-state beam parameters we apply the condition $P_{2}=P_{0}$. Substituting (7) and (8) into (9) we find

$$
P_{0}^{2}+2 U_{1} P_{0}-\frac{\left(-U_{1}-U_{2}\right) \cos ^{2} \eta+U_{1} \sin ^{2} \eta+\left(\frac{2 U_{1} U_{2}}{\eta}-\eta\right) \sin \eta \cos \eta}{\frac{1}{\eta} \sin \eta\left(\cos \eta-\frac{U_{2}}{\eta} \sin \eta\right)}=0
$$

$$
Q(z)=\lambda L / \pi \omega^{2}(z) .
$$

$L$ is the length of the cavity.

Tracing a paraxial light ray through a lenslike medium by using the well-known differential equations for light rays [5], the derivation of matrix elements is straightforward. Further, it has been shown [4] that for the propagation of Gaussian beams in quite general lenslike media (including gain variations) the matrix elements are in which

$$
\eta=L \sqrt{\frac{k_{2}}{k_{0}}}
$$

Since $\eta$ is generally complex, (10) is not directly suitable for calculating the beam parameters $U$ and $Q$. Therefore, other variables are introduced. The constants in (7) can be split into real and imaginary parts 


$$
k=\beta_{0}+i \alpha_{0}+\frac{\beta_{2}}{2} r^{2}+i \frac{\alpha_{2}}{2} r^{2}
$$

so that

$$
\frac{k_{2}}{k_{0}}=-\frac{\beta_{2}+i \alpha_{2}}{\beta_{0}+i \alpha_{0}} \simeq-\frac{\lambda}{2 \pi}\left(\beta_{2}+i \alpha_{2}\right)
$$

provided the gain per wavelength is small $\left(\beta_{0} \gg \alpha_{0}\right)$. Now it follows from (11) that

$$
\eta=\left\{-\frac{\lambda}{2 \pi}\left(\beta_{2} L^{2}+i \alpha_{2} L^{2}\right)\right\}^{1 / 2}
$$

We define further real parameters $\beta$ and $\alpha$

$$
\begin{aligned}
& \beta=\frac{\lambda}{2 \pi} \beta_{2} L^{2} \\
& \alpha=\frac{\lambda}{2 \pi} \alpha_{2} L^{2}
\end{aligned}
$$

which are direct dimensionless measures for the quadratic index- and gain-profiles.

We next introduce the real parameters $p$ and $\phi$

$$
p^{2} e^{2 i \phi}=\beta+i \alpha
$$

so that

$$
\begin{aligned}
p^{2} & =\left\{\beta^{2}+\alpha^{2}\right\}^{1 / 2} \\
\phi & =\frac{1}{2} \tan ^{-1}(\alpha / \beta) .
\end{aligned}
$$

Substituting (16) into (13) we obtain

$$
\eta=i p e^{i \phi} .
$$

Although (13) admits two values for $\eta$, it turns out that they are equivalent and lead to the same final result. ${ }^{1}$ Therefore, we drop the one with the minus sign. We continue with

$$
\eta=i b-a
$$

where

$$
\begin{aligned}
& b=p \cos \phi \\
& a=p \sin \phi .
\end{aligned}
$$

By means of the above substitutions for $\eta$ we can solve (10) and find

$$
P_{0}=-U_{1} \pm i\left\{-U_{1}^{2}-(X+i Y) / N\right\}^{1 / 2}
$$

\footnotetext{
${ }^{1}$ By calculating the angle $\phi$ according to (18), one has to be careful because most computers choose an angle between $-\pi / 2$ and $\pi / 2$, so that for negative values of $\beta$ the calculated angle $\phi$ becomes wrong. The same holds for the calculation of $\tan ^{-1} T / S$ in (29)-(32).
}

where the functions $X, Y$, and $N$ are real. The algebraic derivations are lengthy and tedious. Therefore, we only give the results.

$$
\begin{aligned}
& N=\frac{1}{4 p^{2}}\left(\cosh ^{2} 2 b-\cos ^{2} 2 a\right)+\frac{U_{2}^{2}}{p^{2}}\left(\cosh ^{2} b-\cos ^{2} a\right)^{2} \\
& -\frac{U_{2}}{p^{3}}(\cos \phi \sinh 2 b+\sin \phi \sin 2 a) \\
& \cdot\left(\cosh ^{2} b-\cos ^{2} a\right) \\
& X=\frac{1}{4} \cos 2 \phi\left(\cosh ^{2} 2 b-\cos ^{2} 2 a\right) \\
& -\frac{U_{1}}{4 p}(\cos \phi \sinh 4 b+\sin \phi \sin 4 a) \\
& +\frac{U_{2}}{4 p}\{\cos 2 \phi(\sin \phi \sin 4 a-\cos \phi \sinh 4 b) \\
& -2 \sin 2 \phi(\sin \phi \cos 2 a \sinh 2 b \\
& +\cos \phi \sin 2 a \cosh 2 b)\} \\
& +\frac{U_{1} U_{2}}{4 p^{2}}\{2 \sin 2 \phi \sinh 2 b \sin 2 a \\
& +4 \sin ^{2} \phi \sin ^{2} 2 a+4 \cos ^{2} \phi \sinh ^{2} 2 b \\
& \left.+4 \cos 2 \phi\left(\cosh ^{2} b \sin ^{2} a-\sinh ^{2} b \cos ^{2} a\right)\right\} \\
& +\frac{U_{2}^{2}}{4 p^{2}}\left\{\cos 2 \phi\left(\sinh ^{2} 2 b-\sin ^{2} 2 a\right)\right. \\
& +2 \sin 2 \phi \sinh 2 b \sin 2 a\} \\
& -\frac{U_{1} U_{2}^{2}}{p^{3}}(\cos \phi \sinh 2 b+\sin \phi \sin 2 a) \\
& \cdot\left(\cosh ^{2} b-\cos ^{2} a\right)
\end{aligned}
$$

$$
\begin{aligned}
& Y=\frac{1}{4} \sin 2 \phi\left(\cosh ^{2} 2 b-\cos ^{2} 2 a\right) \\
& +\frac{U_{1}}{4 p}(\cos \phi \sin 4 a-\sin \phi \sinh 4 b) \\
& +\frac{U_{2}}{4 p}\{\sin 2 \phi(\sin \phi \sin 4 a-\cos \phi \sinh 4 b) \\
& +2 \cos 2 \phi(\sin \phi \cos 2 a \sinh 2 b \\
& +\cos \phi \sin 2 a \cosh 2 b)\} \\
& +\frac{U_{1} U_{2}}{4 p^{2}}\{-2 \cos 2 \phi \sinh 2 b \sin 2 a \\
& +2 \sin 2 \phi\left(\sinh ^{2} b-\sin ^{2} a\right) \\
& \left.+4 \sin 2 \phi\left(\cosh ^{2} b \sin ^{2} a-\sinh ^{2} b \cos ^{2} a\right)\right\} \\
& +\frac{U_{2}^{2}}{4 p^{2}}\left\{\sin 2 \phi\left(\sinh ^{2} 2 b-\sin ^{2} 2 a\right)\right. \\
& -2 \cos 2 \phi \sinh 2 b \sin 2 a\} \\
& +\frac{U_{1} U_{2}^{2}}{p^{3}}(\cos \phi \sin 2 a-\sin \phi \sinh 2 b) \\
& \cdot\left(\cosh ^{2} b-\cos ^{2} a\right) \text {. }
\end{aligned}
$$


The two possible solutions for $P_{0}$ given by (23) are not both physically acceptable. It turns out that one solution involves a negative $Q$ value and therefore will be discarded. Which solution given by (23) has to be taken depends on the sign of $-Y / N$. For convenience we introduce $S$ and $T$ given by

$$
\begin{aligned}
& S=-U_{1}^{2}-X / N \\
& T=-Y / N .
\end{aligned}
$$

If $T>0$ we find the following solution

$$
\begin{aligned}
& U=-U_{1}+\left(S^{2}+T^{2}\right)^{1 / 4} \sin \left(\frac{1}{2} \tan ^{-1} T / S\right) \\
& Q=\left(S^{2}+T^{2}\right)^{1 / 4} \cos \left(\frac{1}{2} \tan ^{-1} T / S\right) .
\end{aligned}
$$

If, however, $T<0$ we get

$$
\begin{aligned}
& U=-U_{1}-\left(S^{2}+T^{2}\right)^{1 / 4} \sin \left(\frac{1}{2} \tan ^{-1} T / S\right) \\
& Q=-\left(S^{2}+T^{2}\right)^{1 / 4} \cos \left(\frac{1}{2} \tan ^{-1} T / S\right) .
\end{aligned}
$$

It should be noted that the beam parameters given by (29)-(32) are the values near the cavity mirror with radius $R_{1}$, after reflection.

Although in the above derivations the parameters $Q$ and $U$ are calculated separately, there is a fundamental relationship between them analogous to stream and potential functions in two-dimensional potential theory. The two parameters $Q$ and $U$ are the real and imaginary parts of a function $P_{0}$ given by (10). This means that lines of constant $U$ and lines of constant $Q$ plotted in the $\alpha, \beta$ plane are normal to each other.

\section{Evaluation of Beam Parameters}

Inspecting (23)-(32) we find that as long as some radial gain variation is present, stability of mode structure is obtained independent of mirror configuration. This is in contrast with the description of an empty-space resonator where it is found that stability depends on mirror configuration [1]. If we consider a mirror configuration that is unstable as an empty-space resonator, we find for small values of $\alpha$ the $Q$ value to go to zero with decreasing $\alpha$, which corresponds to an infinitely large spot size, i.e., instability. Therefore, in principle, unstable oscillators may only occur for media with no radial gain variation. Further, we find from (23)-(32) that the beam waist depends only on the absolute value of $\alpha$, whereas the difference between the reciprocal values of the radius of curvature of the wavefront and the mirror curvature changes sign when the sign of the gain variation is reversed.

Thus positive and negative $\alpha$ give rise to stable oscillations. We wish to emphasize this conclusion because it contradicts earlier publications. As an example of erroneous earlier work, [3] states that stable oscillations in a plane-parallel resonator can only be obtained if the gain is highest on the resonator axis. Also in [4] the author ap- parently excluded the possibility of getting stable oscillations for quadratic profiles where the gain increases with distance from the axis. Such a gain profile also leads to stable oscillations, i.e., a finite beam waist, since it leads to a negative radius of curvature of the wavefront. Thus, although one might expect at first glance that a medium whose gain increases with distance from the axis would tend to broaden the beam diameter indefinitely, the present analysis, which accounts for the associated phase changes, shows that wavefront convergence and, hence, a limited beam waist are obtained nevertheless. Before giving numerical examples it is worthwhile to look at the asymptotic behavior for large $\alpha$.

In the limit of very large $\alpha$ values we obtain from (23)-(26)

$$
P_{0_{1 \mathrm{i} m}}= \pm i p\{-i\}^{1 / 2}
$$

for positive $\alpha$, and

$$
P_{0 \mathrm{im}}= \pm i p\{i\}^{1 / 2}
$$

for negative $\alpha$. The asymptotic beam parameters are

$$
Q_{1 \mathrm{im}}=\frac{1}{2} \sqrt{2} p
$$

or

$$
\omega_{\mathrm{im}}=\left\{\frac{4 \lambda}{\pi\left|\alpha_{2}\right|}\right\}^{1 / 4}
$$

for both positive and negative $\alpha$, and

$$
U_{1 \mathrm{~m}}=\mp \frac{1}{2} \sqrt{2} p
$$

for $\alpha= \pm$, or

$$
R_{1 \mathrm{im}}=\mp 2\left\{\frac{\pi}{\lambda\left|\alpha_{2}\right|}\right\}^{1 / 2}
$$

with the minus sign for positive $\alpha$.

In this limit, spot size and curvature of the wavefront become independent of the mirror curvatures.

While the equations describing the beam parameters are derived for laser cavities with arbitrary mirror curvatures, we give numerical data only for configurations with one plane mirror. This type of cavity is often used in practice. By this choice we limit the amount of numerical data to be presented, but we still can demonstrate the general behavior. For this case $U_{2}=0$. From the geometry it is obvious that the beam parameters at the flat mirror can be found from (23)-(32) by just interchanging $U_{1}$ and $U_{2}$, and then letting $U_{2}=0$.

For comparison with the empty-space theory we first caiculate the stability regions of systems having only real refractive-index changes. This is done in Figs. 1 and 2, 


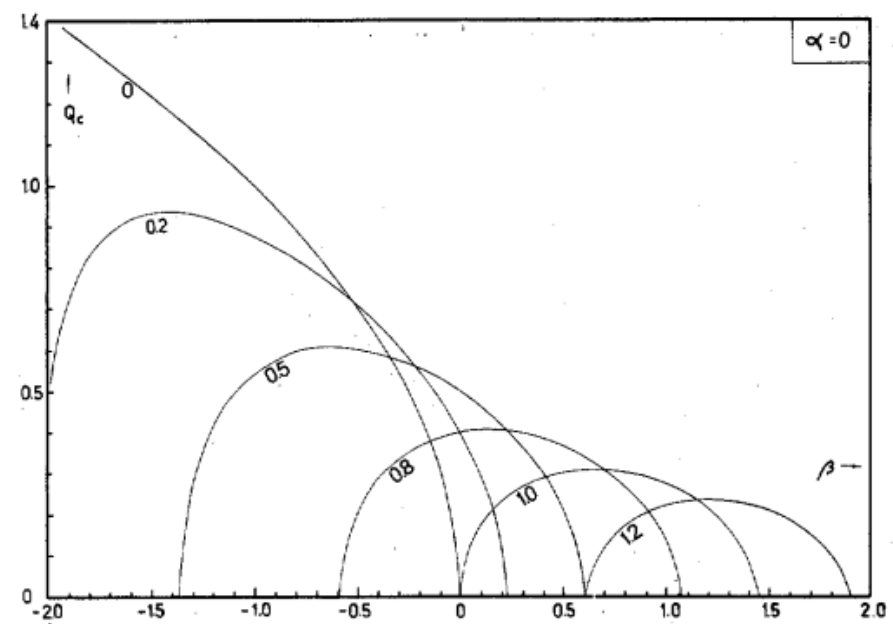

Fig. 1. The beam parameter $Q_{c}=\lambda L / \pi \omega^{2}$ near the curved mirror is calculated for systems with one flat mirror having only refractive-index variations and no gain variations. This is done for the indicated values of $U_{1}=L / R_{1}$.

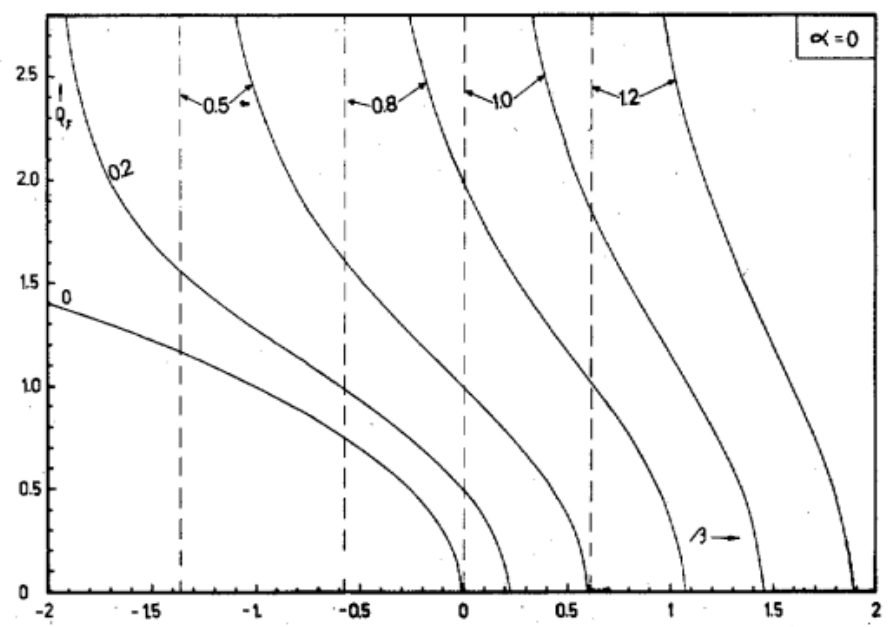

Fig. 2. The beam parameter $Q_{F}$ near the flat mirror is calculated for systems having only refractive-index variations. Different values of $U_{\text {, }}$ are indicated. The dotted lines are the asymptotes.

where the $Q$ values at both mirrors are plotted as a function of $\beta$ for different values of $U_{1}$ and a zero value of $\alpha$. It is seen that for a certain $\beta$ value stable oscillations, i.e., $Q$ larger than zero, can only be obtained for $U_{1}$ values between a lower and upper limit. Further, the $U_{1}$ value for minimum spot size depends on $\beta$. It should be noted that in the absence of gain variations the curvature of the wavefront near a mirror is equal to that of the mirrors. Similarly, in Figs. 3 and 4 we plot the $Q$ values at the two mirrors as a function of $|\alpha|$ for several values of $U_{1}$, but now for $\beta=0$. It is seen that the $Q$ value increases (spot size decreases) with increasing $|\alpha|$ and that the asymptotic value tends to be independent of $U_{\mathrm{i}}$. In Figs. 5 and 6 we plot the curvature of the wavefront $U$ near the two mirrors. This has been done for positive values of $\alpha$. The values of $U$ for negative $\alpha$ can be easily obtained from the fact that the deviation from the reciprocal value of the

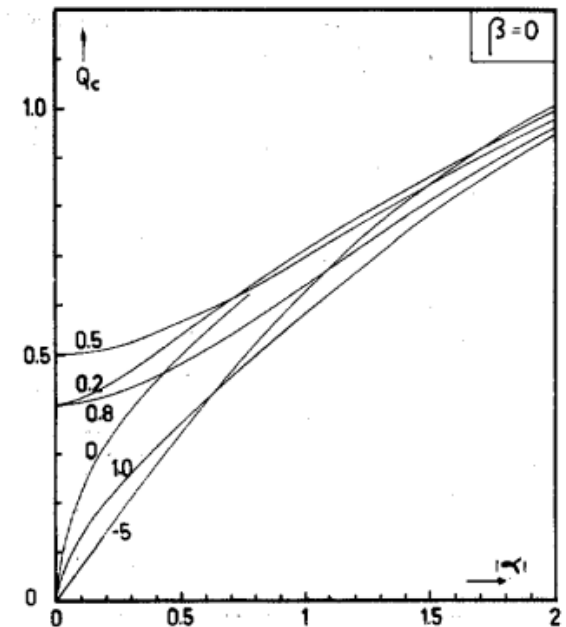

Fig. 3. The beam parameter $Q_{c}$ is calculated for systems with one flat mirror having gain variations but no refractive-index variations. The results depend only on the absolute value of $\alpha$. Values of $U_{1}$ are indicated.

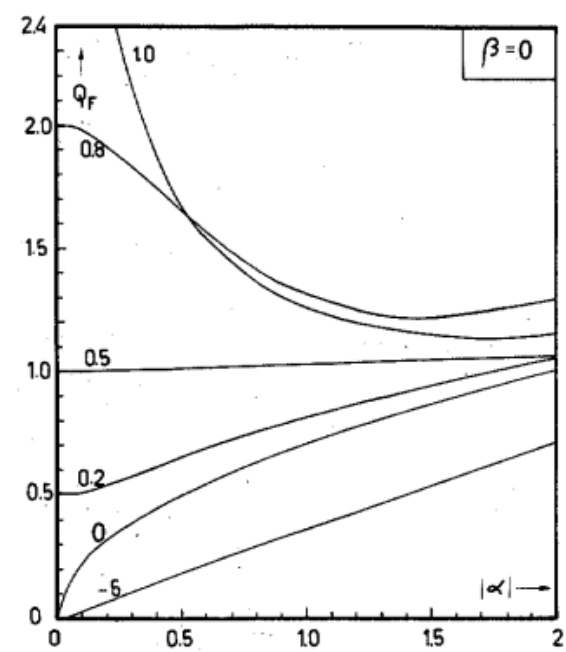

Fig. 4. The beam parameter $Q_{F}$ near the flat mirror is plotted as a function of $\alpha$ for $\beta=0$. The results again depend only on the absolute value of $\alpha$. Values of $U_{1}$ are indicated.

mirror curvature changes sign if $\alpha$ changes sign. This can be formulated as follows. If $\alpha$ is changed into $-\alpha$, the corresponding values of $U$ are related by

$$
U_{-\alpha}=-U_{\alpha}-2 U_{1}
$$

where $U_{-\alpha}$ and $U_{\alpha}$ are, respectively, the $U$ values for negative and positive $\alpha$.

Of most practical use are graphs showing the combined effect of $\alpha$ and $\beta$ to the beam parameters. We calculated iso- $Q$ and iso- $U$ curves for the (semi) confocal system described by $U_{1}=0.5$ and $U_{2}=0$; they are shown in Figs. 7 and 8 , and for the case that both mirrors are flat, in Fig. 9. Fig. 9 shows most clearly the effect of changing the sign of $\alpha$. Because both mirrors are flat, the beam waist and radius of curvature of the wavefront must be independent of position between the mirrors. This means that for 


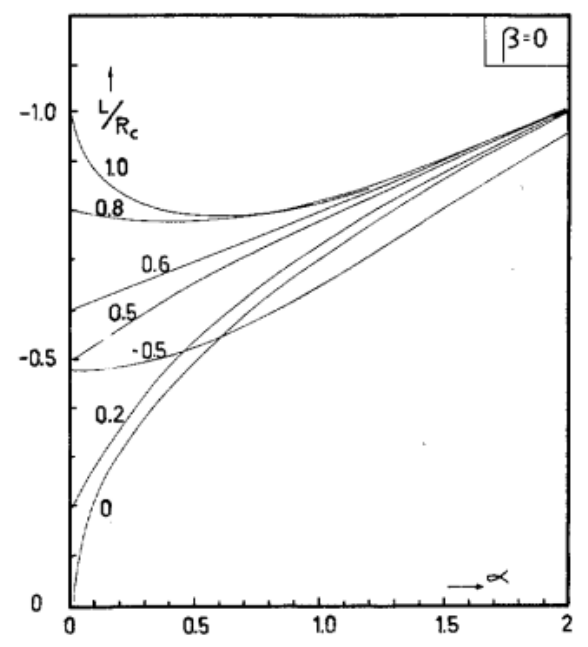

Fig. 5. The graphs show the curvature of the wavefront near the curved mirror of a system having one flat mirror as a function of gain variation (positive values of $\alpha$ ). Various values of $U_{1}$ are indicated. For negative values of the curvature is found by the equation $U_{-\alpha}=-U_{\alpha}-2 U_{1}$.

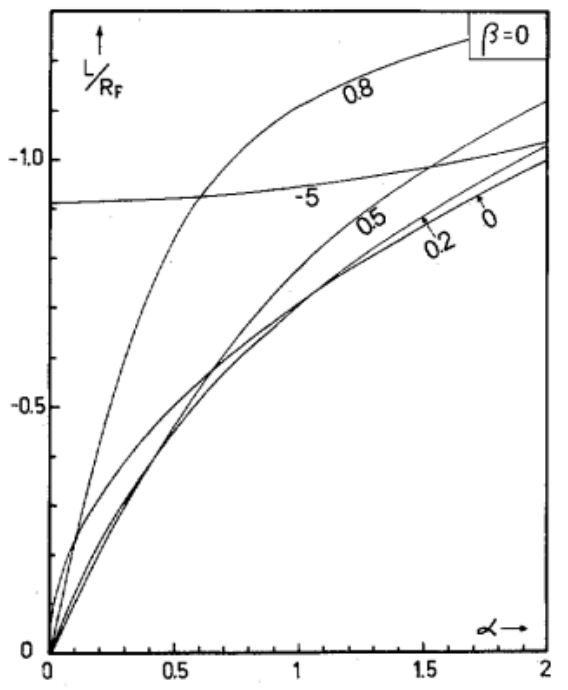

Fig. 6. The curvature of the wavefront near a flat mirror is plotted as a function of positive values of $\alpha$. Values of $U_{1}$ are indicated. For negative $\alpha$ 's one uses the relation $U_{-\alpha}=-U_{\alpha}$.

positive $\alpha$ the beam divergence by the gain is just compensated by the convergence of the wavefront, whereas for negative $\alpha$ the beam convergence by the gain is just compensated by the divergence of the wavefront. For this special case of plane-parallel mirrors a simple relationship between $Q$ and $U$ can be derived,

$$
Q^{2}+U^{2}=p^{2} .
$$

\section{EXPERIMENTAL EXAMPLE}

In Section III we derived the beam parameters for given values of $\alpha$ and $\beta$. As mentioned before, the physical processes that contribute to their values are the heat dissipation by the gas discharge, inhomogeneous gain distribution, and dispersion of the medium. It is outside the scope of the present work to give a general discussion of these processes. Instead, we describe the experimental ex-

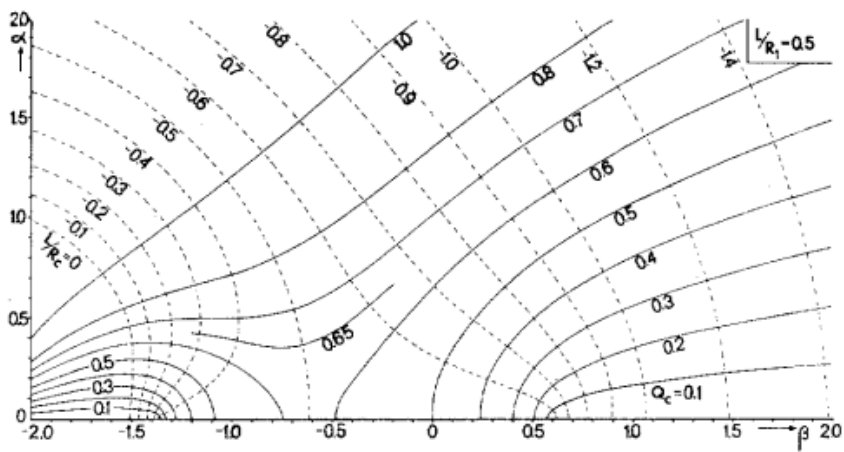

Fig. 7. Lines of constant $Q$ and constant $U$ values evaluated near the curved mirror are shown in the $\alpha, \beta$ plane for a system having one flat mirror and $U_{1}=0.5$. It is seen that the iso- $Q$ and iso- $U$ lines are normal to each other. The results for negative $\alpha$ are similar. Only the parametric value of $U$ has to be changed by $U_{-\alpha}=-U_{\alpha}-2 U_{1}$. The solid lines are iso- $Q$ 's and the dotted lines are iso- $U$ 's.

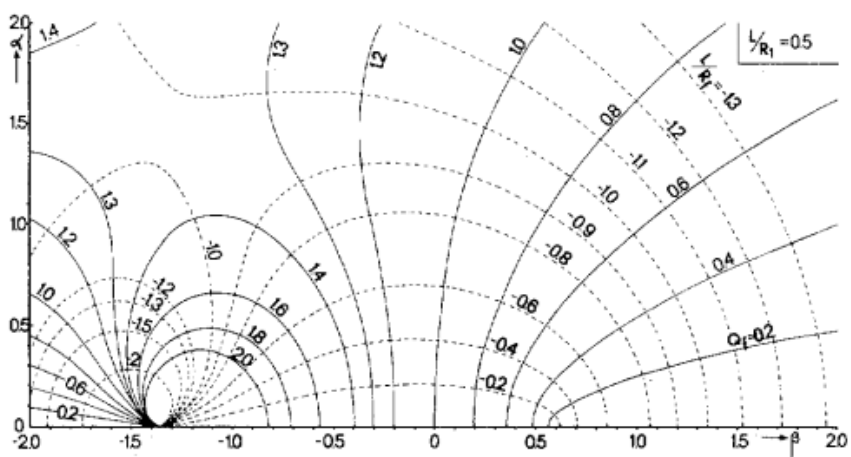

Fig. 8. Lines of constant $Q$ and constant $U$ values evaluated near the flat mirror are shown in the $\alpha, \beta$ plane for $U_{1}=0.5$. The solid lines are iso$Q$ 's and the dotted lines are iso- $U$ 's. The lines are normal to each other. For negative $\alpha$ 's the $U$ 's have to be changed by the relation $U_{-\alpha}=$ $-U_{\alpha}$.

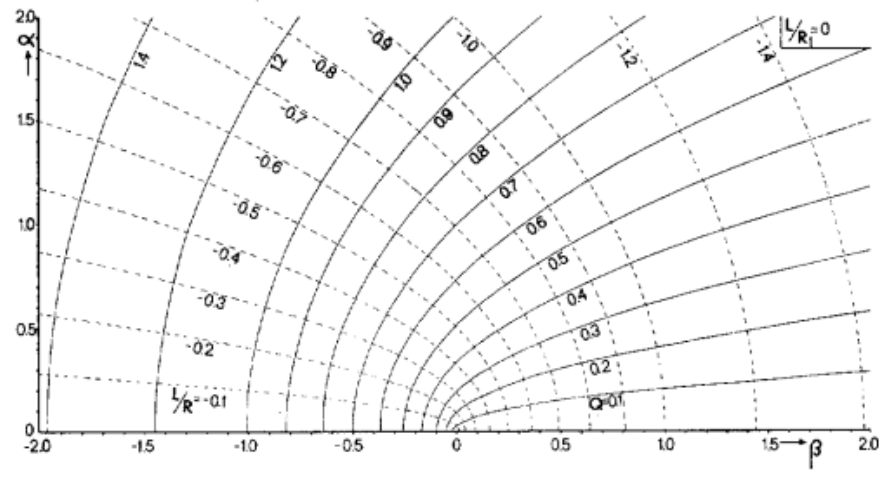

Fig. 9. Lines of constant $Q$ and constant $U$ values valid at any place between two plane-parallel mirrors are shown in the $\alpha, \beta$ plane. The solid lines are iso- $Q$ 's and the dotted lines are iso- $U$ 's. The lines are normal to each other. For negative $\alpha$ 's the $U$ 's have to be interchanged by the relation $U_{\cdot \alpha}=-U_{\alpha}$

ample of a $\mathrm{CO}_{2}$ laser of $380-\mathrm{cm}$ length having a positive column discharge. We just want to show for this example how the parameters $\alpha$ and $\beta$ can be estimated from experimental conditions. The physics of this example is straightforward and requires little discussion.

We intend to show by this example that, first of all, the beam parameter $Q$ indeed deviates considerably from 


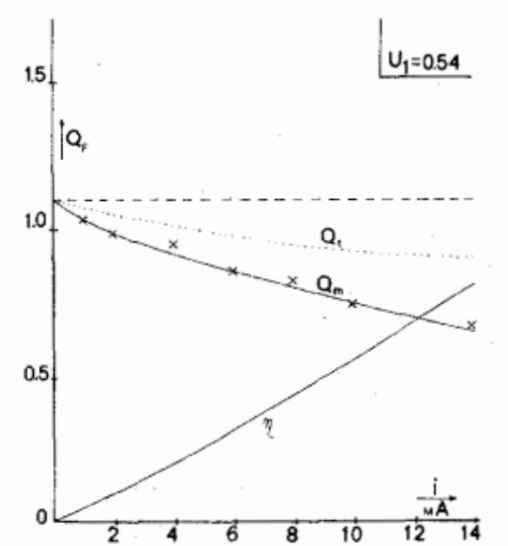

Fig. 10. Experimental observation of $Q$ parameter of a $\mathrm{CO}_{2}$ laser of 380 $\mathrm{cm}$ length and 15 -mm diameter, filled with 1.5-torr $\mathrm{CO}_{2}, 2$-torr $\mathrm{N}_{2}$, and 10 -torr He. Measured values $Q_{m}$ are indicated. The broken horizontal line is the $Q$ value according to the empty-space theory. The dotted line $(Q$.$) represents the value of Q$ that is expected if only thermal effects of the discharge are taken into account. Both, thermal effects and dispersion, the latter caused by the indicated frequency shift $\eta$, account for the experimental observations.

what empty-space theory predicts; secondly, we want to discuss the respective processes; and, finally, evaluate the numerical parameters that account for the experimental observations.

In Fig. 10 we plot experimental observations obtained with a $\mathrm{CO}_{2}$ laser of $380-\mathrm{cm}$ length and $15-\mathrm{mm}$ internal diameter filled with a gas mixture of 1.5-torr $\mathrm{CO}_{2}$, 2-torr $\mathrm{N}_{2}$, and 10-torr $\mathrm{He}$. The cavity has one flat mirror and one totally reflecting with a $7-\mathrm{m}$ radius of curvature. We measured the $Q$ parameters at the plane outcoupling mirror having a reflectivity of about 36 percent. The $Q_{m}$ parameter is measured as a function of the discharge current. The broken horizontal line in the figure represents the value predicted by empty-space theory. In the limit of very small current (about $1 \mathrm{~mA}$ ) this value is indeed observed. At larger currents the beam is still Gaussian but, as is seen, the sizes deviate considerably from the emptyspace theory prediction. Apparently, at small currents the influence of the active medium is negligible.

\section{A. Heat Dissipation of Gas Discharge}

The temperature distribution in a cylindrical symmetric tube can be easily calculated from a given heat production per unit volume and given conductivity of the gas. For a positive column discharge, the mean electron energy is independent of radial position and it is reasonable to assume the heat production is proportional to the product of electron density, which according to simple theory follows a zero-order Bessel function, and gas density. The gas density, in turn, is inversely proportional to the temperature. Using a temperature-dependent heat conductivity for the gas mixture of the $\mathrm{CO}_{2}$ laser, the temperature can be calculated. Further, the refractive index $n$ is related to the gas density by

$$
n=1+k \rho
$$

where $k$ is a constant depending on the gas. The variation of refractive index can be approximated by an expansion in terms of the radial distance from the axis,

$$
n(r)=n_{0}+{ }_{2}^{1} c_{2} r^{2}+\cdots
$$

The quadratic term, which is most important near the axis where the beam intensity is highest, can be easily obtained by differentiating $n$ twice with respect to $r$. Doing this we obtain

$$
c_{2}=\frac{k \rho_{0}{ }^{2} B_{0} d^{4}}{32 I^{2} T_{c}{ }^{3} \lambda_{c}}
$$

$\rho_{0} \quad$ the gas density in the absence of the discharge;

$B_{0}$ the heat dissipation per unit mass density, per unit volume, and per second at the axis of the tube, which is related to the total heat dissipation;

$d$ the tube diameter;

$\lambda_{c}$ the heat conductivity at the axis;

$T_{c} \quad$ the gas temperature at the axis;

$I=\int_{0}^{d / 2} \frac{2 r d r}{T(r)}$

$T(r)$ the temperature at a distance $r$ from the axis.

Going back to (14) we calculate $\beta$ and we find for the thermal part $\beta_{t}$

$$
\beta_{t}=\frac{k \rho_{0}^{2} B_{0} d^{4} L^{2}}{32 I^{2} T_{c}{ }^{3} \lambda_{c}}
$$

If we use this thermal $\beta_{t}$ to compute $Q$ by the theory presented above, we obtain the values denoted by $Q_{t}$ and shown in terms of the dotted line in Fig. 10. It is seen that the thermal effect does not fully account for the experimental behavior.

\section{B. Gain Variations}

In the case of a $\mathrm{CO}_{2}$ laser we are dealing with homogeneous line-broadening. The amplitude gain is given by

$$
\delta=\frac{\frac{1}{2} g}{1+I / I_{0}}
$$

where $g$ is the small signal intensity gain, $I$ is the intensity of the beam, and $I_{s}$ is the saturation parameter.

Considering again a positive column discharge and a lowest order Gaussian beam, gain variations can also be expressed in a power series of the radial distance from the axis. In this expansion the most important term near the axis (where the beam intensity is highest) is quadratic. Thus $\delta=\alpha_{0}+(1 / 2) \alpha_{2} r^{2}+\ldots$. Substituting

$$
\begin{aligned}
& g=g_{0}\left(1+\frac{1}{2} \gamma r^{2}\right) \\
& I_{s}=I_{0 e}\left(1+\frac{1}{2} \beta_{e} r^{2}\right) \\
& I=I_{0} e^{-2 r^{2} / \omega^{2}}
\end{aligned}
$$


into (40) we obtain

$\alpha_{2}=\frac{\frac{1}{2} g_{0}}{1+I_{0} / I_{0 s}}\left\{\gamma+\frac{1}{1+I_{0} / I_{0 s}}-\left(\beta_{\text {s }}+\frac{4}{\omega^{2}}\right) I_{0} / I_{08}\right\}$.

In general $I_{0} / I_{0 s} \gg 1$ and $4 / \omega^{2}>\gamma+\beta_{s}$, so that we approximate $\alpha_{2}$ by

$$
\alpha_{2}=\frac{2 g_{0}}{\omega^{2}} \frac{1}{1+I_{0} / I_{0}}
$$

Going back to (15) we find

$$
\alpha=Q g_{0} L /\left(1+I_{0} / I_{0 s}\right) \simeq-\frac{Q}{2} \ln R_{e}
$$

with $R_{e}$ as the intensity-reflection coefficient of the outcoupling mirror.

\section{Dispersion of the Medium}

Considering a Doppler profile, the variation of refractive index due to the dispersion is given by [6]

$$
\Delta n=\frac{c k}{2 \pi \nu_{0} \sqrt{\pi}} \int_{0}^{n_{1}} \exp \left(x^{2}\right) d x
$$

where $k$ is the gain factor at the frequency $\nu$ for which $\Delta n$ is calculated, $\eta_{1}=2 \sqrt{\ln 2}\left(\nu-\nu_{0}\right) / \Delta \nu_{D}, \nu_{0}$ is the central frequency, and $\Delta \nu_{D}$ is the Doppler linewidth. ${ }^{2}$

The derivation of the associated quadratic profile of refractive index due to the dispersion of the medium is straightforward. In terms of (14) we find

$$
\beta_{d}=\frac{\alpha}{\sqrt{\pi}} \int_{0}^{\eta_{t}} \exp \left(x^{2}\right) d x
$$

Using (44) we obtain for the dispersive part of $\beta$

$$
\beta_{d}=-\frac{Q}{2 \sqrt{\pi}}\left(\ln R_{e}\right) \times \int_{0}^{\eta_{1}} \exp \left(x^{2}\right) d x .
$$

The influence of $\alpha$ on the beam parameter depends strongly on the mirror configuration. In the case of a confocal system its influence is negligible for say $\alpha<0.2$, as can be seen from Figs. 7 and 8. However, for a planeparallel resonator this influence is rather large, as seen in Fig. 9. For the nearly confocal configuration the influence of $\alpha$ is negligible. However, the effect of $\beta_{d}$, as caused by $\alpha$, is rather large and increases with $Q$ as seen by (47). The experimental observations can be explained by taking $\beta$ equal to the sum of $\beta_{t}$ and $\beta_{d}$. If $\eta$ increases with discharge current, i.e., with inversion rate, according to the line indicated in Fig. 10, the observed $Q$ values are explained. However, while these numerical data are consistent and compatible with the experimental results, and hence, tend

\footnotetext{
${ }^{2}$ Note that $\eta$ or $\eta_{1}$ as used in (45)-(47) and in Fig. 10 should not be confused with $\eta$ in (11).
}

to support our interpretation, so far our interpretation is not uniquely proven.

As another result of our experimental work, we found that the oscillating frequency is not close to the central frequency $\nu_{0}$. This is surprising since we deal with a long cavity and homogeneous line broadening and, in addition, many rotational transitions. We intend to explain this behavior in a future contribution where we also plan to show why $\eta$ should increase with discharge current, i.e., with inversion rate. At this point it may suffice to mention that, due to mode competition between axial modes, the favored mode has an increasing positive frequency shift from the central frequency with increasing gain. In a long $\mathrm{CO}_{2}$ laser such as ours, there is always a preferred rotational transition that comes close to this shifted frequency.

\section{CONCLUSION}

The present theory is applicable in the design of long high-gain systems. The length of the tube, due to its quadratic dependence, plays a more important role in the effect than the gain and refractive-index variations themselves. This explains why deviations from the emptyspace theory can be observed for long laser cavities even with moderate index variations. We have seen that the effect of gain and refractive-index variations on the beam parameters may be large so that in order to attain specified operating conditions cavity parameters have to be modified. If, for instance, single-mode operations are required for a medium with gain variation and nonuniform temperature distribution, so that the refractive index also varies, the tube diameter (given by the beam waist) and cavity mirrors for maximum output power can be obtained from the diagrams.

Of particular interest are the iso- $Q$ and iso- $U$ lines in the $\alpha, \beta$ plane. For brevity, only data for the semiconfocal (Fig. 8) and plane-parallel resonator (Fig. 9) were given, but other configurations show a similar behavior. In certain areas of the $\alpha, \beta$ plane, the beam parameters $Q$ and $U$ are very sensitive with respect to small variation of $\alpha$ and $\beta$, in other areas much less so. By means of an experimental example we demonstrated the usefulness of this approach to systems of practical importance. Also the theory possibly contributes to the understanding of experiments with unstable resonators.

\section{REFERENCES}

[1] H. Kogelnik and T. Li, "Laser beams and resonators," Proc. IEEE (Special Joint Issue on Optical Electronics with Applied Optics), vol. 54, pp. 1312-1329, Oct. 1966.

[2] L. W. Casperson and A. Yariv, "The Gaussian mode in optical resonators with a radial gain profile," Appl. Phys. Lett., vol. 12, no. 10, pp. $355-357,1968$.

[3] - , "Gain and dispersion focussing in a high gain laser," Appl. Opt., vol. 12, no. 2, pp. 462-466, 1972.

[4] H. Kogelnik, "On the propagation of Gaussian beams of light through lenslike media including those with a loss or gain variation," Appl. Opt., vol. 4, pp. 1562-1599, Dec. 1965.

[5] M. Born and E. Wolf, Principles of Optics. New York: Pergamon Press, 1959, p. 121.

[6] C. G. B. Garrett, Gas Lasers. New York: McGraw-Hill, 1967, p. 20. 